HUTP-95/A012

March, 1995

\title{
TOPOLOGICAL FIELD THEORY AND PHYSICS
}

\author{
Damiano Anselmi \\ Lyman Laboratory, Harvard University, Cambridge MA 02138, U.S.A.
}

\begin{abstract}
Topological Yang-Mills theory with the Belavin-Polyakov-Schwarz-Tyupkin $S U(2)$ instanton is solved completely, revealing an underlying multi-link intersection theory. Link invariants are also shown to survive the coupling to a certain kind of matter (hyperinstantons). The physical relevance of topological field theory and its invariants is discovered. By embedding topological Yang-Mills theory into pure Yang-Mills theory, it is shown that the topological version TQFT of a quantum field theory QFT allows us to formulate consistently the perturbative expansion of QFT in the topologically nontrivial sectors. In particular, TQFT classifies the set of good measures over the instanton moduli space and solves the inconsistency problems of the previous approaches. The qualitatively new physical implications are pointed out. Link numbers in QCD are related to a non abelian analogoue of the Aharonov-Bohm effect.
\end{abstract}




\section{Introduction}

The idea that in some quantum field theories special correlation functions are exactly calculable dates back to the eighties, when it was realized that supersymmetric theories possess amplitudes ('gaugino condensates' or, in general, 'topological amplitudes') that are independent of the distances between points". A systematic study of topological amplitudes can be pursued in the realm of the so called topological field theories, where every physical amplitude is of this type. Some topological field theories can be produced by a formal procedure, the topological twist [2, 3, 蚆, starting from $\mathrm{N}=2$ supersymmetric theories. Up to now, topological field theories have not been related in a systematic way to their nonsupersymmetric counterparts and their importance for physics has not been identified.

According to Witten [2], the physical amplitudes of topological Yang-Mills theory are the socalled Donaldson invariants [6]. The observables $\mathcal{O}_{\gamma_{i}}$ are interpreted as cocycles of the instanton moduli space $\mathcal{M}$ and are associated to cycles $\gamma_{i}$ of the four-manifold $M$ (Donaldson map). With $M=\mathbb{R}^{4}$ (or $S^{4}$ ), $G=S U(2)$ and unit instanton number, there is no amplitude of this type, since the only nontrivial cycles of $\mathbb{R}^{4}$ are the point (associated to a 4 -form on $\mathcal{M}$ ) and the manifold $M$ itself: the moduli space dimension, which is $8 k-3, k \in \mathbb{Z}$, cannot be saturated with a product of 4 -forms. Nevertheless, in ref. [7] it was explicitly shown that the theory is not empty. The key idea was to integrate the usual observables of topological Yang-Mills theory on contractible closed submanifolds $\gamma_{i}$ of $\mathbb{R}^{4}$, rather than on cycles. The result was that the expectation value of the product of two observables associated to linked submanifolds $\gamma_{1} \subset \mathbb{R}^{4}$ and $\gamma_{2} \subset \mathbb{R}^{4}$ is indeed nonzero. The submanifolds $\gamma_{1}$ and $\gamma_{2}$ are said to be linked if $\gamma_{1}$ is a nontrivial cycle of $\mathbb{R}^{4} \backslash \gamma_{2}$ and $\gamma_{2}$ is a nontrivial cycle of $\mathbb{R}^{4} \backslash \gamma_{1}$. The idea was tested in two cases, namely

$$
<\mathcal{O}_{S^{3}} \cdot \mathcal{O}_{P}>=\chi\left(S^{3}, P\right), \quad<\mathcal{O}_{S^{2}} \cdot \mathcal{O}_{S^{1}}>=\chi\left(S^{2}, S^{1}\right) .
$$

The left hand side denotes the amplitudes as defined in topological Yang-Mills theory. The right hand side denotes the result of the explicit computations, that was interpreted as the link intersection number of the $\gamma_{i}$ 's. I use the symbol $X$ for such a kind of intersection numbers. $X\left(S^{3}, P\right)$ is a step function: zero if the point $P$ is placed outside the 3 -sphere $S^{3} ; 1$ if the point $P$ is placed inside $S^{3} \cdot X\left(S^{2}, S^{1}\right)$ is entirely similar. In ref. [0] correlation functions that do not vanish, although naively expected to, were also found in four dimensional topological gravity.

The considered amplitudes involve non-local observables, related to circles, spheres, Riemann surfaces, 1-knots, 2-knots, etc. In $\mathrm{N}=2$ super Yang-Mills theory the gaugino condensates [1] are average values of local observables. It is interesting to know that amplidutes with nonlocal observables can also be explicitly computed in four dimensions and it would also be desirable to compute similar amplitudes in supersymmetric theories. Moreover, all gaugino condensates are constant amplitudes: step amplitudes have not been found in supersymmetric theories, so

\footnotetext{
${ }^{1}$ For a review and references see [1]. Those results are mainly due to the groups Novikov-Shifman-VainshteinZacharov, Amati-Konishi-Meurice-Rossi-Veneziano, Affleck-Dine-Seiberg.

${ }^{2}$ Recently, nevertheless, it has been proposed in [⿰] that certain amplitudes of topological field theory (Donaldson invariants) can be recovered in ordinary Yang-Mills theory in a suitable limit.
} 
far.

The hidden link-theory contained in topological Yang-Mills theory deserves to be explored in depth. One of the purposes of this paper is to push the analysis of ref. [7] forward, answering some of the questions raised there, in particular identifying completely the mathematical meaning of the unit instanton number sector of the theory. It will be shown that this sector of the theory contains the full set of multilink invariants of closed smooth submanifolds of $\mathbb{R}^{4}$. Multilink intersection theory is defined in section 2 .

In a double linkage $U X V$, one usually writes $U=\partial B$ ( $B$ being called Seifert manifold) and counts the intersections $B \cap V$. There is a well-known integral representation, due to Gauss, of the link number between two 1-knots in $\mathbb{R}^{3}$, namely

$$
U \times V=\frac{1}{4 \pi} \oint_{U} d x^{i} \oint_{V} d y^{j} \varepsilon_{i j k} \partial_{k} \frac{1}{|x-y|} .
$$

Topological field theory provides natural generalizations of this formula. The integral representation of 2-linkages in $\mathbb{R}^{4}$ is known in the literature, the representations of multilikages are new.

In view of the results of []] and the present paper, it seems that the generic ideas according to which $\mathrm{N}=2$ supersymmetric Yang-Mills theory in the ultraviolet is Donaldson theory and the topological twist reorganizes the topological amplitudes of an $\mathrm{N}=2$ theory into a self-consistent topological sub-theory deserves to be reconsidered. Actually, the topological contents of $\mathrm{N}=2$ super Yang-Mills theory ("gaugino condensates"), topological Yang-Mills theory and the socalled "Donaldson theory" appear to be, in general, essentially different and only formally related by the topological twist. Moreover, it will be suggested here that topological YangMills theory is more intrinsically related to ordinary Yang-Mills theory (via a certain topological embedding, see section (4) than to $\mathrm{N}=2$ supersymmetric Yang-Mills theory. Indeed, it is one of the main purposes of this paper to propose and study the role of topological field theory in physics. The topological amplitudes are expected to carry some physical information. Link numbers in QCD should be related to a non abelian analogue of the Aharonov-Bohm effect (which could be in principle detectable).

The organization of the paper is the following. In section 2, multilink intersection theory is defined and tested by computing various amplitudes and by working out integral representations of multilink invariants. In section 3 the so-called hyperinstantons, introduced and studied by Frè and the author in ref.s [4, 8, 9], are used to show that the properties of pure topological Yang-Mills theory survive the coupling to matter (scalar fields, in this case). In section \&, the topological embedding is realized and compared to the usual treatment of collective coordinates. Finally, in section 5 the relation between link numbers and Aharonov-Bohm effect is discussed.

\section{Multilinks}

The aim of this section is to define multilink intersection theory, work out its relation with topological Yang-Mills theory, test this relation and find integral representations of multilink invariants. 
According to the common interpretation, topological field theory deals with intersection theory on the moduli space $\mathcal{M}$ of some instantons on a manifold $M$. This means that the average value of a product of observables $\mathcal{O}_{\gamma_{i}}$ integrated over cycles $\gamma_{i} \subset M$ has an interpretation

$$
<\mathcal{O}_{\gamma_{1}} \cdots \mathcal{O}_{\gamma_{n}}>=\#\left(L_{1}, \cdots, L_{n}\right)
$$

as intersection number (here denoted with \#) of cycles $L_{i} \subset \mathcal{M}$ associated to the $M$-cycles $\gamma_{i}$. The above expression is well-defined and possibly nonzero only when the intersection on the right hand side is a complete intersection, which means

$$
\sum_{i=1}^{n} \operatorname{codim} L_{i}=\operatorname{dim} \mathcal{M}
$$

In that case, the intersection $\cap_{i} L_{i}$ is a discrete set of points and the operation \# counts these points (with a suitable sign assignement that can be defined rigorously).

Now, the results of ref. [7] imply that this is not the whole story about topological field theories in four dimensions, in general. Indeed, link numbers belong to a quite different class of invariants. Nevertheless, one expects that the interpretation of these new topological correlation functions is formally similar to the above one, once one replaces the symbol \# with $X$ and $L_{i}$ with $\gamma_{i}$ themselves. Moreover, $\gamma_{i}$ are closed $M$-submanifolds, but not necessarily $M$-cycles. Thus, we expect to have, instead of (2.1),

$$
<\mathcal{O}_{\gamma_{1}} \cdots \mathcal{O}_{\gamma_{n}}>=X\left(\gamma_{1}, \cdots, \gamma_{n}\right)
$$

and that this expression is possibly nontrivial when some analogue of (2.2) holds.

For simplicity, I assume that none of the $\gamma_{i}$ is $M$ itself. In general, the $\gamma_{i}$ 's wil be compact. However, in the explicit calculations it is sometimes convenient to 'uncompactify' them, for example describing a 2-sphere as a 2-plane.

The above symbolic expressions suggest that one should be able define a suitable concept of multi-linkage and a suitable criterium of complete multilinkage.

Completeness.

A multiple intersection point $P$ among a set of $M$-submanifolds $\gamma_{1}, \ldots \gamma_{n}$ is complete if

$$
\sum_{i=1}^{n} \operatorname{codim} \gamma_{i}=\operatorname{dim} M+1
$$

$P$ is called a complete intersection. $X\left(\gamma_{1}, \ldots \gamma_{n}\right)$ is called a complete multilink intersection form. All situations in which (2.4) does not hold are referred to as incomplete intersections.

In relation (2.4) (as well as in the multilink problem) there is no trace either of $S U(2)$ instantons, or topological Yang-Mills amplitudes. The funny fact is that for $M=\mathbb{R}^{4}$ (or $S^{4}$ ), $\operatorname{dim} M+1$ equals the dimension of the moduli space of $S U(2)$ instantons on $M$ with unit instanton number. Condition (2.4) is clearly satisfied by the amplitudes (1.1). Indeed, from the field theoretical point of view, (2.4) is nothing but the requirement that the ghost number anomaly should be compensated by the sum of the ghost numbers of the observables. 
For $n=2$, (2.4) can be written in the form $\operatorname{dim} \gamma_{1}+\operatorname{dim} \gamma_{2}=\operatorname{dim} M-1$, which is the usual rule for 2-linkages. However, this form is not suitable for the multilink generalization: the correct expression is (2.4).

\section{Multi-linkage.}

The idea of multilinkage is the following. Consider the amplitude (2.3). One wants to deform the $\gamma_{i}$ 's smoothly in $\mathbb{R}^{4}$ in orther to unlink them or contract them to points: in practice, to move them very far from one another. In doing this, two things can happen:

i) a proper subset of the $\gamma_{i}$ 's intersect in some point or some locus of points. This is an incomplete intersection and is valued 0 . The reason for this is that, when the $\gamma_{i}$ 's satisfy (2.4), then no proper subset of the $\gamma_{i}$ 's can satisfy an analogous relation and intersect completely. For example, two 2-spheres $S^{2}$ and $S^{2 \prime}$ can be intersected and superposed without problems.

ii) in the movement, it is necessary to cross complete intersections, i.e. points in which all the $\gamma_{i}$ 's intersect contemporarily. Each of these points contributes with one unit. The multilink intersection number is the (algebraic) counting of these points. It is easy to see that, in a generic situation, this is the counting of a discrete number of points.

The rigorous definition of the signs of each contribution is encoded in the explicit integral formulæ that will be derived from topological field theory.

The following property holds:

$$
X\left(\gamma_{1}, \ldots \gamma_{i}, \gamma_{i+1}, \ldots \gamma_{n}\right)=(-1)^{\operatorname{codim} \gamma_{i} \cdot \operatorname{codim} \gamma_{i+1}} X\left(\gamma_{1}, \ldots \gamma_{i+1}, \gamma_{i}, \ldots \gamma_{n}\right)
$$

that means that one has to take the order in which the $\gamma_{i}$ 's are listed into account.

Considering the example $\times\left(S^{3}, S^{3^{\prime}}, S^{1}\right)$ of a triple linkage with two 3-spheres $S^{3}$ and $S^{3^{\prime}}$ and one circle $S^{1}$, depicted in the figure, one can easily work out alternative definitions of the multilink intersection points. The picture is at fixed time $t=0$ : the 3 -spheres appear as 2-spheres at fixed time. The circle $S^{1}$ is at $t \equiv 0 . S^{1}$ intersects $S^{3}$ in the points $P$ and $S$ and $S^{3^{\prime}}$ in $R$ and $Q$. The intersection among the two 3 -spheres is a 2 -sphere $S^{2}$. At $t=0$ such an intersection appears as a circle $C$. $C$ is the boundary of a surface $D$ (the shadowed region in the picture), that intersects $S^{1}$ in a point $T$. The counting of the points $T$ obtained in this way with appropriate signs gives the amplitude. Describing the set of points that have to be counted does not seem so difficult, but it seems nontrivial to assign appropriate signs to them. Topological Yang-Mills theory already contains the correct prescription. 


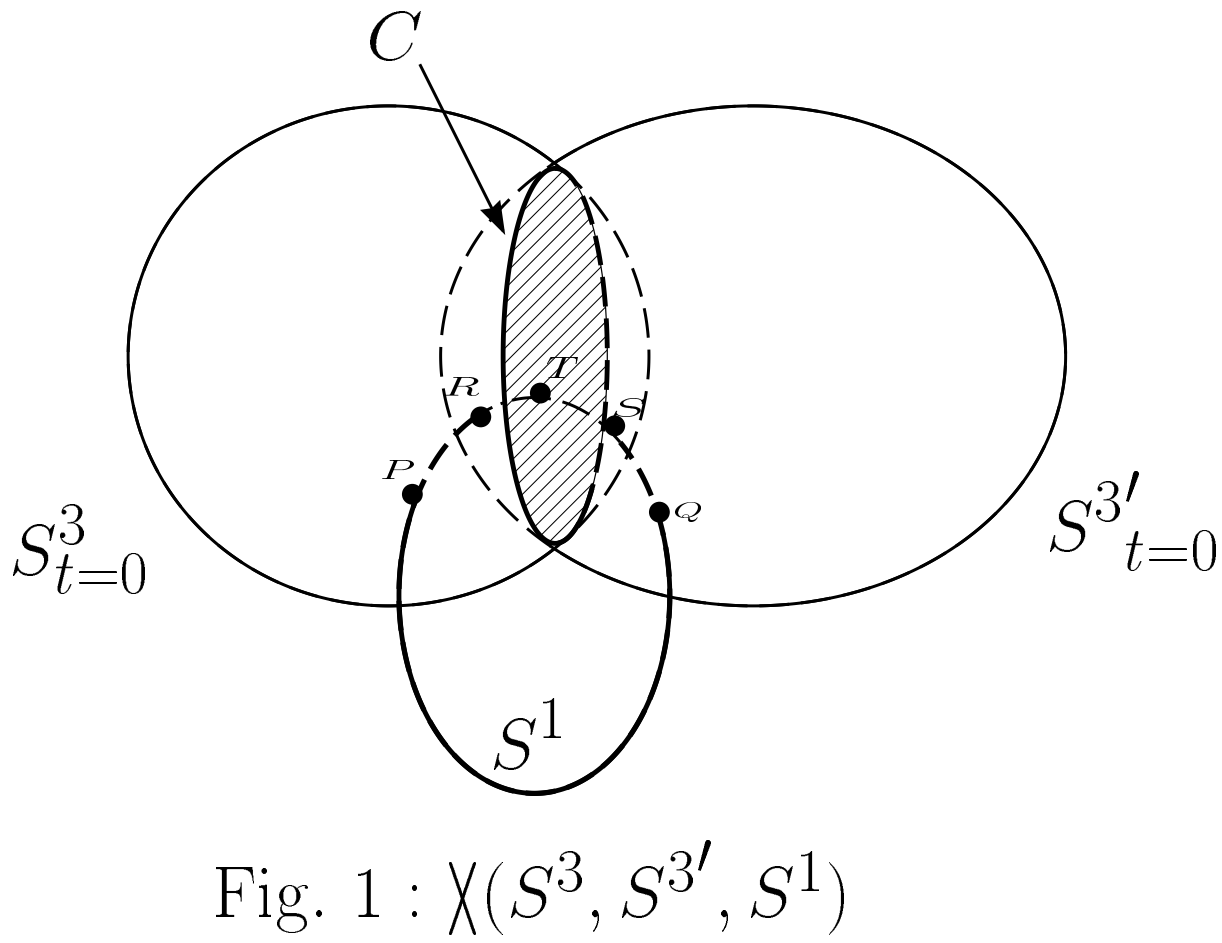

To show that the above construction is meaningful, one has to prove that it does not depend on the chosen couple of $\gamma_{i}$ 's one starts with. So, let us consider, now, the following alternative possibility. This time, we start by considering the intersection between $S^{3}$ and $S^{1}$, which is represented by the two points $P$ and $S . P$ and $S$ are the boundary of a segment $\overline{P S} . \overline{P S}$ meets $S^{3}$ in a point $N$ (not shown in the picture). The amplitude can also be described as the counting of such points.

A third equivalent description is the following. Let $\beta_{1}$ be such that $\partial \beta_{1}=\gamma_{1}$. Then, consider the intersection among $\beta_{1}$ and the other $\gamma_{i}$ 's, $i=2, \ldots n$. This is in general a discrete set of points and counting them gives the amplitude.

Proposition. Multilink intersection theory is the solution to topological Yang-Mills theory with $G=S U(2), M=\mathbb{R}^{4}$ and unit instanton number.

I am now going to check the above proposition in various cases of multilink intersections and derive their integral representations. It is useful to report here the explicit solution of the theory, as it was elaborated in ref. [7], to which the reader is referred for the details of the derivation (see also formula (4.4)). The solution is encoded into the generating expressions

$$
\begin{aligned}
& \hat{\mathcal{Q}}=\frac{1}{16 \pi^{2}} \hat{F}^{a} \hat{F}^{a}=\frac{1}{4 \pi^{2}} \frac{\rho^{3}}{D^{4}}\left[\rho d V\left(x-x_{0}\right)-4 d \rho \wedge\left(x-x_{0}\right)^{\mu} d \sigma_{\mu}\left(x-x_{0}\right)\right]=\frac{1}{16 \pi^{2}} \hat{d} \hat{C}, \\
& \hat{C}=\hat{A}^{a} \hat{F}^{a}-\frac{1}{6} \varepsilon_{a b c} \hat{A}^{a} \hat{A}^{b} \hat{A}^{c}=\frac{4}{3} \frac{1}{D^{3}}\left[3 \rho^{2}+\left(x-x_{0}\right)^{2}\right]\left(x-x_{0}\right)^{\mu} d \sigma_{\mu}\left(x-x_{0}\right) .
\end{aligned}
$$

$\left(\rho, x_{0}\right) \in \mathcal{M}=(0, \infty) \otimes \mathbb{R}^{4}$ are the five moduli, while $x \in M=\mathbb{R}^{4}$ and $D=\rho^{2}+\left(x-x_{0}\right)^{2}$. 
$F^{a}=d A^{a}+\frac{1}{2} \varepsilon^{a}{ }_{b c} A^{b} A^{c}$ denotes the field strength. $\hat{F}^{a}=F^{a}+\psi_{0}^{a}+\phi_{0}^{a}$ and $\hat{A}^{a}=A^{a}+C_{0}^{a}$ are the relevant BRST extensions. Finally, $d V(x)=d x^{\mu} d \sigma_{\mu}(x), d \sigma_{\mu}(x)=\varepsilon_{\mu \nu \rho \sigma} d x^{\nu} d x^{\rho} d x^{\sigma}$ and $\hat{d}=d+s, s$ being the BRST operator

$$
s=d m^{i} \frac{\partial}{\partial m^{i}}=d \rho \frac{\partial}{\partial \rho}+d x_{0}^{\mu} \frac{\partial}{\partial x_{0}^{\mu}} .
$$

The observables are

$$
\mathcal{O}_{\gamma}^{(n)}=\int_{\gamma} \hat{\mathcal{Q}}^{n}
$$

It was noticed in [7] that the amplitudes are "automatically" normalized correctly by the factor $\frac{1}{16 \pi^{2}}$ appearing in (2.6). This property gives an intrinsic and concrete meaning to formal concepts and set-ups like the 'BRST extension' (see [10, 3, , 4, 8]). The notation of ref. [7] is strictly followed in the sequel, with the only difference that the observables $\mathcal{O}$ and the corresponding $\mathcal{M}$-differential forms are defined ab-initio with the correct normalization factor $\frac{1}{16 \pi^{2}}$.

Any topological amplitude can be written as an integral over the boundary of the moduli space $\mathcal{M}$. For $S U(2)$ instantons on $S^{4}$ such a boundary corresponds to $\rho=0$, while for instantons on $\mathbb{R}^{4}$ the boundary possesses more components $\left(\rho \rightarrow \infty\right.$ and $\left.x_{0} \rightarrow \infty\right)$. Nevertheless, these extra components never contribute to the amplitudes computed in ref. [7], confirming that one can safely extend the results to topological Yang-Mills theory on $S^{4}$. In this case, however, one should imagine that a puncture is placed at infinity. In the appendix to this section it is proved on general grounds, that the $\partial \mathcal{M}$-component $x_{0} \rightarrow \infty$ never contributes. Knowing this a priori is useful to simplify the computations, since in many cases, it is convenient to 'uncompactify' some submanifolds $\gamma_{i}$ and it is not correct to check the $x_{0} \rightarrow \infty$ component after having uncompactified. I shall comment on the vanishing of the $\rho \rightarrow \infty$ terms along with the computations.

The $\rho \rightarrow 0$ limit of $(2.6)$ can be done easily, using the property

$$
\lim _{\rho \rightarrow 0} \frac{\rho^{4}}{\left(\rho^{2}+x^{2}\right)^{4}}=\frac{\pi^{2}}{6} \delta(x)
$$

so that on $\partial \mathcal{M}(d \rho$ being also zero)

$$
\hat{\mathcal{Q}}(x) \rightarrow \frac{1}{4 !} \delta\left(x-x_{0}\right) d V\left(x-x_{0}\right)=-\frac{1}{4 ! \pi^{2}} \hat{d} \partial_{\mu} \frac{1}{\left(x-x_{0}\right)^{2}} d \sigma_{\mu}\left(x-x_{0}\right) .
$$

In practice, everything is encoded into these very simple expressions, although it is (2.6) that makes any computation meaningful. Some properties, that are not visible from (2.10) will follow immediately from (2.6). Check, for example, the end of subsection 2.2 .

Now, we are ready to begin the computations. First (Example 1) the integral representation of $X\left(\gamma_{1}, \gamma_{2}\right)$ is derived from topological field theory. Then, we proceed by studying genuine multilink invariants and extracting their integral representations. The calculation of the first multilink intersection number (Example 2) is done in full detail, the other computations being simply scketched. 


\subsection{Evaluations}

Example 1. Integral representation of $X\left(\gamma_{1}, \gamma_{2}\right)$.

Let us consider two submanifolds $\gamma_{1}, \gamma_{2} \subset \mathbb{R}^{4}$, of dimensions 1 and 2 , respectively. Let the corresponding observables be $\mathcal{O}_{\gamma_{1}}=\omega_{\gamma_{1}}^{(3)}=d \Omega_{\gamma_{1}}^{(2)}$ and $\mathcal{O}_{\gamma_{2}}=\omega_{\gamma_{2}}^{(2)}=d \Omega_{\gamma_{2}}^{(1)}$. We have

$$
X\left(\gamma_{1}, \gamma_{2}\right)=<\mathcal{O}_{\gamma_{1}} \cdot \mathcal{O}_{\gamma_{2}}>=\int_{\mathcal{M}} \omega_{\gamma_{1}}^{(3)} \wedge \omega_{\gamma_{2}}^{(2)}=\int_{\partial \mathcal{M}} \Omega_{\gamma_{1}}^{(2)} \wedge \omega_{\gamma_{2}}^{(2)}
$$

$\Omega_{\gamma_{1}}^{(2)}$ and $\omega_{\gamma_{2}}^{(2)}$ can be easily written down from (2.6). We have thus

$$
X\left(\gamma_{1}, \gamma_{2}\right)=-\frac{3}{2 \pi^{4}} \int_{\gamma_{1}} d x^{\mu} \int_{\gamma_{2}} d y^{\nu} d y^{\rho} \lim _{\rho \rightarrow 0} \int_{\mathbb{R}^{4}} d^{4} x_{0} \frac{\varepsilon_{\mu \nu \rho \sigma}\left(x-x_{0}\right)^{\sigma} \rho^{4}\left[3 \rho^{2}+\left(x-x_{0}\right)^{2}\right]}{\left[\rho^{2}+\left(x-x_{0}\right)^{2}\right]^{3}\left[\rho^{2}+\left(y-x_{0}\right)^{2}\right]^{4}} .
$$

It is convenient to write

$$
X\left(\gamma_{1}, \gamma_{2}\right)=\int_{\gamma_{1}} d x^{\mu} \int_{\gamma_{2}} d y^{\nu} d y^{\rho} \varepsilon_{\mu \nu \rho \sigma} V^{\sigma}(x-y),
$$

where

$$
V^{\sigma}(z)=-\frac{3}{2 \pi^{4}} \lim _{\rho \rightarrow 0} \int_{\mathbb{R}^{4}} d^{4} x_{0} \frac{\left(z-x_{0}\right)^{\sigma} \rho^{4}\left[3 \rho^{2}+\left(z-x_{0}\right)^{2}\right]}{\left[\rho^{2}+\left(z-x_{0}\right)^{2}\right]^{3}\left(\rho^{2}+x_{0}^{2}\right)^{4}}=-\frac{1}{4 \pi^{2}} \frac{z^{\sigma}}{|z|^{4}} .
$$

The form of the last expression follows from dimensional considerations. The constant $-\frac{1}{4 \pi^{2}}$ is determined after rescaling all quantities by $\rho$ and applying the dominated convergence theorem, or, more quickly, but less rigorously, by using (2.9). We conclude

$$
X\left(\gamma_{1}, \gamma_{2}\right)=\frac{1}{8 \pi^{2}} \int_{\gamma_{1}} d x^{\mu} \int_{\gamma_{2}} d y^{\nu} d y^{\rho} \varepsilon_{\mu \nu \rho \sigma} \partial_{\sigma} \frac{1}{(x-y)^{2}}
$$

which is the desired expression. Notice the appearance of the Green function $\frac{1}{(x-y)^{2}}$, which is singular precisely on the complete intersection points $x=y$. Of course, (2.15) contains (1.2): writing $\mathbb{R}^{4}$ as $\mathbb{R}^{3} \otimes \mathbb{R}$ and taking $U=\gamma_{1} \subset \mathbb{R}^{3}, \gamma_{2}=V \otimes \mathbb{R}, V \subset \mathbb{R}^{3}$, (2.15) gives back (1.2).

Example 2. $\times\left(S^{3}, S^{3^{\prime}}, S^{1}\right)$.

In the first example of multilinkage, I consider a triple intersection among two 3-spheres and one circle (see also Fig. 1). The 3 -spheres will be 'uncompactified' to $\mathbb{R}^{3}$ 's, this simplifying a bit the calculation. Precisely, I choose

$$
S^{3}=\mathbb{R}^{2} \times \mathbb{R} \times\{0\}, \quad S^{3^{\prime}}=\mathbb{R}^{2} \times\{\bar{x}\} \times \mathbb{R}, \quad S_{r}^{1}=\{(0,0)\} \times C_{r},
$$

$C_{r}$ denoting a circle of radius $r$ placed in the last $\mathbb{R}^{2}$ and centered in the origin. The above submanifolds of $\mathbb{R}^{4}$ are indeed multilinked according to the general definition. $S^{3}$ and $S^{3^{\prime}}$ intersect in the 'time' axis (which, by convention, is the first one, while the other axis are $x$, $y$ and $z$, in the order) and in the $x$-axis. Such intersections are irrelevant, because incomplete. The circle $S^{1}$ is at fixed time $t=0$ and winds around the $x$-axis. It intersects $S^{3}$ in a couple of points on the $y$-axis and $S^{3^{\prime}}$ in a couple of points on the $z$-axis. These intersections are also incomplete. Now, if $r>\bar{x}$, when one wants to unlink the three objects, it is necessary to cross precisely one complete (triple) intersection point. Thus, we predict that the corresponding 
topological amplitude is equal to one. Instead, if $r<\bar{x}$, the unlinking procedure goes on safely and we predict a zero amplitude.

I want to compute

$$
\mathcal{A}=<\mathcal{O}_{S^{3}} \cdot \mathcal{O}_{S^{3^{\prime}}} \cdot \mathcal{O}_{S_{r}^{1}}>=\int_{\mathcal{M}} \omega^{(1)} \wedge \omega^{(1)^{\prime}} \wedge \omega_{r}^{(3)}
$$

Using the first of $(\sqrt{2.6})$, it is easy to verify that the $\mathcal{M}$-1-forms corresponding to the first two observables are

$$
\omega^{(1)}=\int_{S^{3}} \hat{Q}=\frac{3}{4} \frac{\rho^{3}\left[\rho d x_{0}^{4}-x_{0}^{4} d \rho\right]}{\left[\rho^{2}+\left(x_{0}^{4}\right)^{2}\right]^{\frac{5}{2}}}, \quad \omega^{(1)^{\prime}}=\int_{S^{3^{\prime}}} \hat{Q}=\frac{3}{4} \frac{\rho^{3}\left[\rho d x_{0}^{3}-\left(x_{0}^{3}\right) d \rho\right]}{\left[\rho^{2}+\left(x_{0}^{3}-\bar{x}\right)^{2}\right]^{\frac{5}{2}}} .
$$

An immediate check shows that these differential forms are closed, as it must be.

Instead, in order to write down $\omega_{r}^{(3)}$, it is convenient to use the second of (2.6), to express $\omega_{r}^{(3)}$ as $d \Omega_{r}^{(2)}$, and $\mathcal{A}$ as $\int_{\partial \mathcal{M}} \omega^{(1)} \wedge \omega^{(1)^{\prime}} \wedge \Omega_{r}^{(2)}$. One finds

$$
\Omega_{r}^{(2)}=\frac{1}{4 \pi^{2}} \int_{C_{r}} \frac{3 \rho^{2}+\left(x_{0}^{1}\right)^{2}+\left(x_{0}^{2}\right)^{2}+\left(\mathbf{x}-\mathbf{x}_{0}\right)^{2}}{\left[\rho^{2}+\left(x_{0}^{1}\right)^{2}+\left(x_{0}^{2}\right)^{2}+\left(\mathbf{x}-\mathbf{x}_{0}\right)^{2}\right]^{3}}\left(x-x_{0}\right)^{\mu} d x^{\nu} \varepsilon_{\mu \nu \rho \sigma} d x_{0}^{\rho} d x_{0}^{\sigma},
$$

the bold-face denoting 2 -vectors in the plane generated by the $y$-axis and the $z$-axis.

We know that the only component of the boundary $\partial \mathcal{M}$ that contributes is the one for $\rho \rightarrow 0$. So, we can put $d \rho=0$ and take the limit $\rho \rightarrow 0$. Let us also choose $\bar{x}=0$, for now. We have

$$
\mathcal{A}=\lim _{\rho \rightarrow 0} \frac{9 \rho^{8}}{32 \pi^{2}} \int_{\mathbb{R}^{4}} d^{4} x_{0} \int_{0}^{2 \pi} d \theta \frac{r\left(r-x_{4} \cos \theta\right)\left(3 \rho^{2}+x_{0}^{2}+r^{2}-2 r x_{4} \cos \theta\right)}{\left[\rho^{2}+\left(x_{0}^{4}\right)^{2}\right]^{\frac{5}{2}}\left[\rho^{2}+\left(x_{0}^{3}\right)^{2}\right]^{\frac{5}{2}}\left(\rho^{2}+x_{0}^{2}+r^{2}-2 r x_{4} \cos \theta\right)^{3}},
$$

where $x_{4}=\sqrt{\left(x_{0}^{3}\right)^{2}+\left(x_{0}^{4}\right)^{2}}$. Now, rescaling all quantities by $\rho$, redefining $r$ as $\frac{r}{\rho}$ and integrating over $x_{0}^{1}, x_{0}^{2}$ and $\theta$ one easily arrives at

$$
\mathcal{A}=\lim _{r \rightarrow \infty} \frac{9}{32 \pi} \int_{-\infty}^{\infty} d x_{0}^{4} \int_{-\infty}^{\infty} d x_{0}^{3} \frac{\varphi\left(x_{4}, r\right)}{\left[1+\left(x_{0}^{4}\right)^{2}\right]^{\frac{5}{2}}\left[1+\left(x_{0}^{3}\right)^{2}\right]^{\frac{5}{2}}}
$$

where

$$
\varphi\left(x_{4}, r\right)=\pi+\pi \frac{\left(r^{2}-x_{4}^{2}\right)^{3}+3\left(r^{4}-x_{4}^{4}-x_{4}^{2}\right)+r^{2}-1}{\left[\left(r^{2}-x_{4}^{2}\right)^{2}+2\left(r^{2}+x_{4}^{2}\right)+1\right]^{\frac{3}{2}}} .
$$

The function $\varphi\left(x_{4}, r\right)$ tends to zero for $x_{4} \rightarrow \infty$, to $2 \pi$ for $r \rightarrow \infty$, is continuous and bounded. So, by the dominated convergence theorem, we can exchange the limit and the integration, finally obtaining

$$
\mathcal{A}=\frac{9}{16}\left(\int_{-\infty}^{\infty} \frac{d y}{\left(1+y^{2}\right)^{\frac{5}{2}}}\right)^{2}=1
$$

confirming the expectation. Moreover, $\varphi\left(x_{4}, 0\right) \equiv 0$. So, for $r \rightarrow 0$, which corresponds to $\rho \rightarrow \infty$, we have a zero result, confirming that the $\partial \mathcal{M}$-component $\rho \rightarrow \infty$ does not contribute.

Taking $\bar{x} \neq 0$, there are two quantities that, after the rescaling by $\rho$, should tend to infinity: the rescaled $r$ and the rescaled $\bar{x}$. The order according to which these limits should to be taken 
is dictated by which one of the inequalities $r>\bar{x}$ and $r<\bar{x}$ is true. In the first case, everything goes on as before, but in the second case one gets zero. We conclude

$$
\mathcal{A}(r, \bar{x})=\frac{1}{2}(1+H(r-\bar{x})) .
$$

where $H(x)=1$ for $x>0$ and $H(x)=-1$ for $x<0$ : the amplitude is a step function. Finally, one can check that for $r=\bar{x}$ the result is $\frac{1}{2}$, so that we can define $H(0)=0$.

Example 3. $\times\left(S_{(1)}^{3}, S_{(2)}^{3}, S_{(3)}^{3}, S_{r}^{2}\right)$.

In this example, the 3 -spheres will be described by $\mathbb{R}^{3}$ 's, at $x=0, y=0$ and $z=0$, respectively. The corresponding $\mathcal{M}$-forms are the analogues of (2.18). Thus, we have a triple intersection, which is the entire time axis at $x=y=z=0$. The 2 -sphere $S_{r}^{2}$ will be placed at fixed time $t=0$ and centered in the origin of the 3-space generated by the $x, y$ and $z$-axis. Vectors in such a three space will be written in boldface. It is obvious that one cannot unlink the four objects without meeting one complete intersection point. Using the second of (2.6) we write $\mathcal{A}=\int_{\partial \mathcal{M}} \prod_{i=1}^{3} \omega_{i}^{(1)} \Omega_{r}^{(1)}$, with $\omega_{r}^{(2)}=d \Omega_{r}^{(1)}$ and

$$
\Omega_{r}^{(1)}=\frac{1}{4 \pi^{2}} \int_{S_{r}^{2}} \frac{\left[3 \rho^{2}+\left(x_{0}^{1}\right)^{2}+\left(\mathbf{x}-\mathbf{x}_{0}\right)^{2}\right]\left(x-x_{0}\right)^{\mu} \varepsilon_{\mu \nu \rho \sigma} d x^{\nu} d x^{\rho} d x_{0}^{\sigma}}{\left[\rho^{2}+\left(x_{0}^{1}\right)^{2}+\left(\mathbf{x}-\mathbf{x}_{0}\right)^{2}\right]^{3}} .
$$

Thus, after rescaling all quantities by $\rho$ and integrating ove $x_{0}^{1}$, one easily arrives at

$$
\mathcal{A}=\lim _{r \rightarrow \infty} \frac{3^{3}}{2 \cdot 4^{3}} \int \prod_{i=2}^{4} d x_{0}^{i} \int_{0}^{\pi} d \theta \frac{r^{2} \sin \theta\left(r-\left|\mathbf{x}_{0}\right| \cos \theta\right)}{\prod_{i=2}^{4}\left[1+\left(x_{0}^{i}\right)^{2}\right]^{\frac{5}{2}}\left(r^{2}+\left|\mathbf{x}_{0}\right|^{2}-2 r\left|\mathbf{x}_{0}\right| \cos \theta\right)^{\frac{3}{2}}} .
$$

Finally, taking the limit $r \rightarrow \infty$, one finds

$$
\mathcal{A}=\frac{3^{3}}{4^{3}}\left(\int_{-\infty}^{\infty} \frac{d y}{\left(1+y^{2}\right)^{\frac{5}{2}}}\right)^{3}=1 .
$$

Example 4. X $\left(S_{(1)}^{3}, S_{(2)}^{3}, S_{(3)}^{3}, S_{(4)}^{3}, S_{(5)}^{3}\right)$.

Now we want to check the predictions with a 5 -tuple linkage among 3 -spheres. The first four 3 -spheres $S_{(i)}^{3} i=1, \ldots 4$ will be in fact $\mathbb{R}^{3}$ 's, at $t=\bar{t}, x=\bar{x}, y=\bar{y}$ and $z=\bar{z}$, respectively. They intersect in the point $\bar{x}=(\bar{t}, \bar{x}, \bar{y}, \bar{z}) \in \mathbb{R}^{4}$. This is a 4-intersection and so does not contribute, according to the general rules: in the case at hand a complete intersection is a 5 -intersection. The fifth 3 -sphere $S_{(5)}^{3}$ (which will be really a compact sphere, the radius being $r$ ) is chosen to surround the origin. Consequently, if $r>|\bar{x}|$ the unlinking process necessarily meets a 5 -tuple intersection point and the amplitude $\mathcal{A}=\int_{\mathcal{M}} \prod_{i=1}^{5} \omega_{i}^{(1)}$ is expected to be equal to one. If, instead, $r<\bar{x}$, the unlinking process finds no obstacle and the result is zero.

The expressions of $\omega_{i}^{(1)}, i=1, \ldots 4$ are easily read from (2.18), while, using some other results of [7], see formulæ (4.21) and (4.24) there, one can write $\omega_{5}^{(1)}=d f$, where $f$ is a function that tends to $\frac{1}{2}\left(1+H\left(r-x_{0}\right)\right)$ for $\rho \rightarrow 0$. Consequently, we have

$$
\mathcal{A}=\lim _{\rho \rightarrow 0} \frac{3^{4}}{4^{4}} \int_{\mathbb{R}^{4}} \frac{1}{2}\left(1+H\left(r-\left|x_{0}+\bar{x}\right|\right)\right) \frac{\rho^{16} d^{4} x_{0}}{\prod_{i=1}^{4}\left[\rho^{2}+\left(x_{0}^{i}\right)^{2}\right]^{\frac{5}{2}}} .
$$


Rescaling every quantity by $\rho$ as usual, one gets

$$
\mathcal{A}=\frac{3^{4}}{4^{4}}\left(\int_{-\infty}^{\infty} \frac{d y}{\left(1+y^{2}\right)^{\frac{5}{2}}}\right)^{4} \frac{1}{2}(1+H(r-\bar{x}))=\frac{1}{2}(1+H(r-\bar{x})),
$$

as desired.

\subsection{Integral representations of multilink invariants}

The purpose of this subsection is to extract the integral representations of multilink invariants provided by the instanton. I focus on $X\left(\gamma_{3}, \gamma_{3}^{\prime}, \gamma_{1}\right)$. It is easy to see that the integral representation of $X\left(\gamma_{3}, \gamma_{3}^{\prime}, \gamma_{1}\right)=\int_{\mathcal{M}} \omega_{\gamma_{3}}^{(1)} \wedge \omega_{\gamma_{3}^{\prime}}^{(1)} \wedge \omega_{\gamma_{1}}^{(3)}=\int_{\partial \mathcal{M}} \omega_{\gamma_{3}}^{(1)} \wedge \omega_{\gamma_{3}^{\prime}}^{(1)} \wedge \Omega_{\gamma_{1}}^{(2)}$ provided by the instanton can be written as

$$
X\left(\gamma_{3}, \gamma_{3}^{\prime}, \gamma_{1}\right)=\int_{\gamma_{3}} \int_{\gamma_{3}^{\prime}} \int_{\gamma_{1}} d \sigma(x) \cdot d y d \sigma\left(x^{\prime}\right) \cdot V\left(x, x^{\prime}, y\right)-d \sigma\left(x^{\prime}\right) \cdot d y d \sigma(x) \cdot V\left(x, x^{\prime}, y\right),
$$

where $V^{\mu}\left(x, x^{\prime}, y\right)=\frac{\partial}{\partial y^{\mu}} v\left(x, x^{\prime}, y\right)$. Here, $v\left(x, x^{\prime}, y\right)$ is a kind of "three-body Green function". Its expression is

$$
v\left(x, x^{\prime}, y\right)=\frac{1}{4 \pi^{6}} \lim _{\rho \rightarrow 0} \rho^{8} \int_{\mathbb{R}^{4}} d^{4} x_{0} \frac{1}{\left[\rho^{2}+\left(y-x_{0}\right)^{2}\right]\left[\rho^{2}+\left(x-x_{0}\right)^{2}\right]^{4}\left[\rho^{2}+\left(x^{\prime}-x_{0}\right)^{2}\right]^{4}} .
$$

$v\left(x, x^{\prime}, y\right)$ should be regarded as a distribution and can be easily determined using (2.9). Alternatively, it is convenient to study $\square_{y} v\left(x, x^{\prime}, y\right) \equiv v_{y}\left(x, x^{\prime}, y\right)$, picking up a test function $\varphi(z, t)$, $z=x-y, t=x^{\prime}-y$, and acting on it with $v_{y}$. We have

$$
\begin{aligned}
v_{y}(\varphi) & =\int v_{y}\left(x, x^{\prime}, y\right) \varphi(z, t) d z d t \\
& =-\frac{2}{\pi^{6}} \lim _{\rho \rightarrow 0} \int \frac{\rho^{10} \varphi(z, t) d z d t d x_{0}}{\left(\rho^{2}+x_{0}^{2}\right)^{3}\left[\rho^{2}+\left(z-x_{0}\right)^{2}\right]^{4}\left[\rho^{2}+\left(t-x_{0}\right)^{2}\right]^{4}}=-\frac{1}{36} \varphi(0,0),
\end{aligned}
$$

after rescaling $z, t$ and $x_{0}$ by $\rho$, as usual. We conclude

$$
v_{y}\left(x, x^{\prime}, y\right)=-\frac{1}{36} \delta(x-y) \delta\left(x^{\prime}-y\right), \quad v\left(x, x^{\prime}, y\right)=\frac{1}{36 \pi^{2}} \frac{\delta\left(x-x^{\prime}\right)}{\left(x-2 y+x^{\prime}\right)^{2}} .
$$

The final expression of the triple link number is thus

$$
X\left(\gamma_{3}, \gamma_{3}^{\prime}, \gamma_{1}\right)=\frac{1}{36 \pi^{2}} \int_{\gamma_{3}} d \sigma^{\mu}(x) \int_{\gamma_{3}^{\prime}} d \sigma^{\nu}\left(x^{\prime}\right) \int_{\gamma_{1}} d y^{\rho} \delta\left(x-x^{\prime}\right) \frac{\partial}{\partial y^{\sigma}} \frac{\left(\delta_{\mu \rho} \delta_{\nu \sigma}-\delta_{\mu \sigma} \delta_{\nu \rho}\right)}{\left(x-2 y+x^{\prime}\right)^{2}}
$$

This expression, as well as (2.33), is in complete agreement with the multilink idea. The delta function projects onto the instersection of two submanifolds, the rest counts the links with the third manifold. Notice that the Green function $v\left(x, x^{\prime}, y\right)$ is not symmetric in $x, x^{\prime}$ and $y$, but keeps trace of the choice of the two submanifolds that are intersected. This choice corresponds to the choice of which differential form is converted from $\omega_{\gamma}^{(n)}$ to $\Omega_{\gamma}^{(n-1)}$ (where $\omega_{\gamma}^{(n)}=d \Omega_{\gamma}^{(n-1)}$ ) when passing from the integral over $\mathcal{M}$ to the integral over the boundary $\partial \mathcal{M}$. 
So, the instanton provides an easy way to prove that the result is independent of the choice of the intersected submanidolds, although this independence is not apparent in formula (2.34). The above expression was found converting $\omega_{\gamma_{1}}^{(3)}$ to $\Omega_{\gamma_{1}}^{(2)}$. Alternatively, doing the same work with $\gamma_{3}$, instead of $\gamma_{1}$, one finds the equivalent integral representation

$$
X\left(\gamma_{3}, \gamma_{3}^{\prime}, \gamma_{1}\right)=\frac{1}{36 \pi^{2}} \int_{\gamma_{3}} d \sigma^{\mu}(x) \int_{\gamma_{3}^{\prime}} d \sigma^{\nu}\left(x^{\prime}\right) \int_{\gamma_{1}} d y^{\nu} \delta\left(y-x^{\prime}\right) \frac{\partial}{\partial x^{\mu}} \frac{1}{\left(y-2 x+x^{\prime}\right)^{2}}
$$

In all the other cases, Green functions and integral representations can be worked out similarly.

\subsection{Open problems}

We have estabilished that multilink intersection theory is the solution to topological YangMills theory on $\mathbb{R}^{4}$ (or $S^{4}$ ) with $G=S U(2)$ and unit instanton number. Open problems concern the interpretation (and calculation) of nonvanishing amplitudes for the other instanton numbers [11, 12], as well as for other gauge groups $G$ and manifolds $M$. For $G=S U(N)$ and unit instanton number, the amplitudes are the same, since the instanton is the same. When $G=S U(3)$ the formal dimension of the moduli space is $12 k-8$, while for $S U(2)$ it is $8 k-3$. For $k=1$, one has 4 instead of 5 . This is because, embedding the $S U(2)$ instanton in the first three generators of $S U(3)$, there is room for an antighost zero mode (the constant, which is indeed meaningful on $S^{4}$, but not on $\mathbb{R}^{4}$ ) associated to the eight $S U(3)$ generator. A reasonable way to define amplitudes (denoted with $\ll \ldots \gg$ ) with selection rule 4 instead of 5 can be

$$
\ll \mathcal{O}_{1} \cdots \mathcal{O}_{n} \gg=\lim _{r \rightarrow \infty}<\mathcal{O}_{S_{r}^{3}} \cdot \mathcal{O}_{1} \cdots \mathcal{O}_{n}>
$$

In this way, one has, for example, using the first of (1.1),

$$
\ll \operatorname{tr}\left[\phi^{2}\right](x) \gg=\lim _{r \rightarrow \infty}<\mathcal{O}_{S_{r}^{3}} \cdot \operatorname{tr}\left[\phi^{2}\right](x)>=1,
$$

since the infinitely large 3 -sphere necessarily contains the point $x$. Formula (2.37) is an example of a recursion relation between invariants for different $N$ 's, perhaps a particular case of a much richer set of recursion relations.

As far as higher instanton numbers are concerned and $G=S U(2)$, we can make the following comments. For generic $k$ a very simple amplitude is $\left\langle\mathcal{O}_{S_{r}^{3}} \cdot \prod_{i=1}^{2 k-1} \operatorname{tr}\left[\phi^{2}\right]\left(x_{i}\right)>[\mathbb{7}]\right.$. A possible meaning of this amplitude is that it counts the number of points $x_{i}$ that are placed inside $S_{r}^{3}$. Apart from this very simple case, however, it is not so easy to identify the meaning of the more complicated amplitudes. Take, for example, $k=3$ and $<\prod_{i=1}^{3} \int_{\gamma_{i}} \mathcal{Q}^{2}>, \gamma_{i}, i=1,2,3$, being three circles in $\mathbb{R}^{4}$. This should be something like the generalized link number of three circles $\gamma_{i}$. If this has a meaning, the meaning should be nontrivial. One can expect that there is a canonical way of associating a 2-sphere or, in general, a 2-knot to a couple of circles and that the amplitude is the linking number between the 2 -knot and the third circle. Moreover, this result shoul be independent of the choice of the initial couple of circles. The present knowledge on 2-knots, however, does not allow us to say whether this description makes any sense or should 
be discarded tout court. It can be taken for granted, anyway, that uncovering the meaning of the amplitudes of this theory is a source of insight for mathematics itself.

Finally, it is also interesting to know whether the properties described so far survive the coupling to matter. A positive answer will be given in the next section, where explicit examples of the so-called hyperinstantons introduced in ref. [4, 8, 9] by Frè and the author will be studied.

\subsection{Appendix: $x_{0} \rightarrow \infty$ does not contribute}

Here I prove that the $x_{0} \rightarrow \infty \partial \mathcal{M}$-component does not contribute to the topological amplitudes. The amplitude is always written as $\int_{\mathcal{M}} \prod_{i=1}^{n} \omega_{\gamma_{i}}=\int_{\partial \mathcal{M}} \Omega_{\gamma_{1}} \prod_{i=2}^{n} \omega_{\gamma_{i}}$ where $\omega_{\gamma_{1}}=d \Omega_{\gamma_{1}}$. Since the $\gamma_{i}$ 's are assumed to be compact, there exists an $R$ such that the 3 -sphere of radius $R$ centered in the origin contains any $\gamma_{i}$. Then, it is easy to prove, from (2.6) and (2.8), that

$$
\omega_{\gamma_{i}} \sim \frac{\rho^{3} R^{d_{i}}\left(\rho d^{4-d_{i}} x_{0}+x_{0} d \rho d^{3-d_{i}} x_{0}\right)}{\left(\rho^{2}+x_{0}^{2}\right)^{4}}, \quad \Omega_{\gamma_{i}} \sim \frac{R^{d_{i}}\left(3 \rho^{2}+x_{0}^{2}\right) x_{0} d^{3-d_{i}} x_{0}}{\left(\rho^{2}+x_{0}^{2}\right)^{3}}, \quad \text { for } x_{0} \rightarrow \infty,
$$

where $d_{i}=\operatorname{dim} \gamma_{i}$. Due to the fact that $\sum_{i=1}^{n} \operatorname{codim} \gamma_{i}=5$, we have $\sum_{i=1}^{n} d_{i}=4 n-5$. Since $n \geq 2$, then $\sum_{i=1}^{n} d_{i} \geq 3$. Let us assume $n=2$, which is the worst case. Then, the $x_{0} \rightarrow \infty$ contribution is of the form

$$
\int_{0}^{\infty} d \rho \frac{R^{3}\left(3 \rho^{2}+x_{0}^{2}\right) x_{0}^{5} \rho^{3}}{\left(\rho^{2}+x_{0}^{2}\right)^{7}} \sim \frac{R^{3}}{x_{0}^{3}} \rightarrow 0
$$

as expected. Note that if we first uncompactified some of the $\gamma_{i}$ 's $(R \rightarrow \infty)$ and then took the limit $x_{0} \rightarrow \infty$ (which is incorrect), we would find problems.

\section{Matter coupling}

In this section, the coupling to matter (scalar fields) is examined. The ideas introduced in ref.s [4, 8, 9] by Fré and the author are used extensively. Scalars can possess very interesting instantons (called hyperinstantons), that can be coupled to gravitational instantons [4, \&], as well as Yang-Mills instantons [9]. Here we take isospin 1/2 scalar fields in the background of the Belavin et al. [13] instanton. The coupling constant $g$ is set to 1 , for simplicity. Hyperistantons are described by the lagrangian (I convert to the Euclidean signature with respect to ref. 9])

$$
\frac{\mathcal{L}}{\sqrt{g}}=\frac{1}{2} g^{\mu \nu} h_{i j} \mathcal{D}_{\mu} q^{i} \mathcal{D}_{\nu} q^{j}+\frac{1}{4 \beta} F_{\mu \nu}^{a} F_{\mu \nu}^{a}+\frac{\beta}{2} \mathcal{P}_{a}^{u} \mathcal{P}_{a}^{u}
$$

A parameter $\beta$ has been introduced for future use. This lagrangian is the bosonic piece of an $\mathrm{N}=2$ lagrangian in a special case suggested by the topological twist [9]. $\mathcal{L}$ can be written as

$$
\begin{aligned}
\frac{\mathcal{L}}{\sqrt{g}} & =\frac{1}{8} g^{\mu \nu} h_{i j}\left(\mathcal{D}_{\mu} q^{i}-\Lambda^{u v}\left(j_{u}\right)_{\mu}{ }^{\rho} \mathcal{D}_{\rho} q^{k}\left(J_{v}\right)_{k}{ }^{i}\right)\left(\mathcal{D}_{\nu} q^{j}-\Lambda^{s t}\left(j_{s}\right)_{\nu}{ }^{\sigma} \mathcal{D}_{\sigma} q^{l}\left(J_{t}\right)_{l}{ }^{j}\right) \\
& +\frac{1}{2 \beta}\left(F_{\mu \nu}^{-a}+\frac{\beta}{2} \Lambda_{u v} I_{\mu \nu}^{u} \mathcal{P}_{a}^{v}\right)^{2}+\frac{1}{8 \beta} F^{a} F^{a}+\frac{1}{8} \Lambda_{u v} \Theta^{u} \hat{\Omega}^{v} .
\end{aligned}
$$


$\Lambda^{u v}$ can be any $S O(3)$ matrix. See [9] for the remaining notation, that will be in any case explained along with the discussion. The instanton configurations are

$$
F_{\mu \nu}^{-a}+\frac{\beta}{2} \Lambda_{u v} I_{\mu \nu}^{u} \mathcal{P}_{a}^{v}=0, \quad \mathcal{D}_{\mu} q^{i}-\Lambda^{u v}\left(j_{u}\right)_{\mu}{ }^{\nu} \mathcal{D}_{\nu} q^{j}\left(J_{v}\right)_{j}{ }^{i}=0
$$

I shall take $\beta \rightarrow 0$, so that the first equation reduces to the usual equation of Yang-Mills instantons, solved by the first of (4.4). The other equation is invariant and defines the hyperinstantons in the gauge-instanton background (or gauged triholomorphic maps, according to the mathematical interpretation worked out in [8, 9]). The topological properties should be independent of $\beta$, since changing $\beta$ should be a continuous deformation of the instanton equation. It could happen, nevertheless, that some value of $\beta$ is not reachable continuously, but I do not enter into these problems here. After factorizing $\exp \left(-\frac{1}{8 \beta} F^{a} F^{a}\right)$ away, the limit $\beta \rightarrow 0$ in the Lagrangian $\mathcal{L}(3.2)$ can be thought as a kind of Landau gauge (indeed, from the point of view of topological field theory, the instanton conditions are simply the gauge-fixing of the topological symmetry). Therefore, the $\beta=0$ solutions are hyperinstantons in the Landau gauge.

To be explicit, in the case at hand $j_{u}=J_{u}=I^{u}=-\bar{\eta}_{u}, \bar{\eta}_{u}$ denoting the anti-self-dual 't Hooft symbols [14], $\Theta^{u}=d x^{t} I^{u} d x, \Omega^{u}=d q^{t} I^{u} d q, \hat{\Omega}^{u}=\mathcal{D} q^{t} I^{u} \mathcal{D} q+F^{a} \mathcal{P}_{a}^{u}, \mathcal{D}_{\mu} q^{i}=\partial_{\mu} q^{i}+$ $\frac{1}{2} A_{\mu}^{a}\left(\bar{I}_{a}\right)^{i j} q_{j}, \mathcal{P}_{a}^{u}=\frac{1}{2} q^{t} I^{u} \bar{I}^{a} q, \bar{I}_{a}=-\eta_{a}$. One has $d \Theta^{u}=d \Omega^{u}=d \hat{\Omega}^{u}=0$. The index $i=1, \ldots 4$ goes over the real components of the isospin $1 / 2$ representation.

Consider the following configuration

$$
q^{i}(x)=\frac{\left(x-x_{0}\right)^{i}}{\rho \sqrt{\rho^{2}+\left(x-x_{0}\right)^{2}}}=\frac{\left(x-x_{0}\right)^{i}}{\rho \sqrt{D}} .
$$

One can check that $\mathcal{D}_{\mu} q^{i}=\frac{\delta_{\mu}^{i} \rho}{D^{3 / 2}}$. The second equation of (3.3) gives $\Lambda=\Lambda^{t}$ and $\operatorname{tr} \Lambda=-1$. We choose $\Lambda=\operatorname{diag}(1,-1,-1)$. In this way, the above scalar field configuration, which was known only as a solution to the equation $D_{\mu} D^{\mu} q^{i}=0$ [14], is also a hyperinstanton]. This remark allows us to use the machinery developped in ref. [ [, 8, 6]. Notice the crucial power $1 / \rho$ in (3.4).

Now, one wants to elaborate the specific observables of the above scalar configuration and check whether they share the properties of pure Yang-Mills theory. The observables are related, via descent equations, to a topological number, called hyperinstanton number, identified in [8, 9] as the last term of (3.2), namelyf

$$
\operatorname{Hn}=\frac{1}{4 \pi^{2}} \int_{M} \Lambda_{u v} \Theta^{u} \wedge \hat{\Omega}^{v},
$$

\footnotetext{
${ }^{3}$ As a matter of fact, it is also a solution to certain vortex equations, related to $\mathrm{N}=1$ supersymmetry in the same way as the hyperinstanton equations are related to $\mathrm{N}=2$ supersymmetry.

${ }^{4}$ As pointed out in [9], eqs. (3.3) reduce to Witten's monopole equations [15] when $G=U(1)$ and the manifold of the scalars $q$ is flat (in ref.s 8, 8 the $q$-manifold can be a generic almost quaternionic manifold). The hyperinstanton number, firstly introduced in [8], here plays a crucial role, but it seems that in [15] there is no analogue of it.
} 
where $\hat{\Omega}^{u}=d \hat{\omega}^{u}$ and $\hat{\omega}^{u}=q^{t} I_{u} d q+A^{a} \mathcal{P}_{a}^{u}$. One can check that $\mathrm{Hn}=1$ for the solution (3.4). The instantons of the coupled theories are thus classified by two integer numbers: the usual instanton number and the hyperinstanton number.

One could put an arbitrary constant $v$ in front of $q^{i}$, however such a parameter should not be considered as a modulus: the BRST variation of $v$ should be zero, otherwise $\mathrm{Hn}=v^{2}$ would not be BRST invariant (see section 6 of ref. [7] for an analogous case in topological abelian YangMills theory coupled to topological gravity). The eventual $v$-integration is made meaningful by the exponential factor $\mathrm{e}^{-\mathrm{Hn}}$. In practice, we can suppress the parameter $v$.

The desired observables are provided by the descent equations associated to $\mathrm{Hn}$ in the usual way 10, 3, 8]. To begin with, let us consider

$$
\mathcal{O}_{S_{r}^{3}}=\frac{1}{4 \pi^{2}} \int_{S_{r}^{3}} \Lambda_{u v} \Theta^{u}\left(2 I_{i j}^{v} \mathcal{D} q^{i} \xi^{j}+\psi_{0}^{a} \mathcal{P}_{a}^{v}\right)=\frac{1}{4 \pi^{2}} s \int_{S_{r}^{3}} \Lambda_{u v} \Theta^{u} \hat{\omega}^{v}=s f\left(r, \rho, x_{0}\right),
$$

where $s$ is the BRST operator (2.7) and $\xi^{i}$ is defined by $s q^{i}=\xi^{i}-C_{0}^{a} k_{a}^{i}, k_{a}^{i}(q)=\frac{1}{2}\left(\bar{I}_{a}\right)^{i j} q_{j}$. $\psi_{0}^{a}=\psi_{0 \mu}^{a} d x^{\mu}$ and $C_{0}^{a}$ are given in (4.4). One finds

$$
f\left(r, \rho, x_{0}\right)=\frac{2}{\pi} \int_{0}^{\pi} \frac{r^{3}\left(r-x_{0} \cos \theta\right) \sin ^{2} \theta d \theta}{\left(\rho^{2}+r^{2}+x_{0}^{2}-2 r x_{0} \cos \theta\right)^{2}} .
$$

$f$ tends to 1 for $r \rightarrow \infty$, i.e. to the hyperinstanton number Hn. A generic amplitude $\mathcal{A}=<$ $\mathcal{O}_{S_{r}^{3}} \cdot \prod_{i} \mathcal{O}_{\gamma_{i}}>$ can be written as $\int_{\partial \mathcal{M}} f\left(r, \rho, x_{0}\right) \prod_{i} \omega_{\gamma_{i}}$. So, what matters is the limit of $f$ for $\rho \rightarrow 0$. This turns out to be the familiar step function

$$
\lim _{\rho \rightarrow 0} f\left(r, \rho, x_{0}\right)=\frac{1}{2}\left(1+H\left(r-x_{0}\right)\right),
$$

precisely as in the case of pure Yang-Mills theory, but now coming out of very different field configurations (hyperinstantons) and observables related to a different topological number (hyperinstanton number).

While the Yang-Mills instanton number generates $\mathcal{M}$-differential forms of any degree from 1 to 4 (compare with equations (2.6) and (2.8) for $n=1$ ), the hyperinstanton number (3.5) generates only 1-forms and 2-forms, obtained by integrating its descendants over 3- and 2dimensional $M$-submanifolds, respectively. We have just checked that in the first case the pure Yang-Mills result is reproduced. To conclude, we check the same thing in the second case, integrating over the 2-plane $S^{2}=\left\{\mathbf{x}_{2}\right\} \otimes \mathbb{R}^{\prime 2}$ :

$$
\mathcal{O}_{S^{2}}=\omega_{\mathbf{x}_{2}}^{(2)}=d \Omega_{\mathbf{x}_{2}}^{(1)}, \quad \Omega_{\mathbf{x}_{2}}^{(1)}=\frac{1}{4 \pi^{2}} \int_{S^{2}} \Lambda_{u v} \Theta^{u} \omega_{(0,1)}^{v},
$$

$\omega_{(0,1)}^{u}$ being the first descendant of $\hat{\omega}^{u}$. One finds

$$
\xi^{i}=-\frac{\rho d x_{0}^{i}}{D^{3 / 2}}-\frac{\left(x-x_{0}\right)^{i}\left[2 \rho^{2}+\left(x-x_{0}\right)^{2}\right] d \rho}{\rho^{2} D^{3 / 2}}, \quad \omega_{(0,1)}^{u}=q^{t} I_{u} \xi=-\frac{\left(x-x_{0}\right)^{t} I^{u} d x_{0}}{D^{2}} .
$$

Using the $I^{u}$-basis of ref. [9], one has

$$
\Omega_{\mathbf{x}_{2}}^{(1)}=\frac{1}{2 \pi} \frac{\left(x_{2}^{0}-x_{0}^{0}\right) d x_{0}^{1}-\left(x_{2}^{1}-x_{0}^{1}\right) d x_{0}^{0}}{\rho^{2}+\left(\mathbf{x}_{2}-\mathbf{x}_{0}\right)^{2}} .
$$


The pure Yang-Mills analogue of this expression is written in formula (4.41) of ref. [7]. The two expressions are indeed different. However, when inserted into an amplitude, for example, $<\mathcal{O}_{S^{2}} \cdot \mathcal{O}_{S^{1}}>=\chi\left(S^{2}, S^{1}\right)$, only the $\rho \rightarrow 0$ limit matters and both expressions have the same $\rho \rightarrow 0$ limit, apart from an immaterial sign and the normalization (the overal factor $\frac{1}{16 \pi^{2}}$, not introduced in [7] from the begining). So, again, the coupling to matter agrees with the pure theory, normalization included.

\section{Topological embedding}

The purpose of this section and the next one is to study the relevance of link invariants and topological field theory for physics. In the end of the day the properties found in ref. [7] and in the previous sections are properties of very special solutions to the field equations of pure QCD. It is hard to believe that such properties have no relation with physics.

The first aim is to embed topological Yang-Mills theory into ordinary Yang-Mills theory and show in what limit (and for what amplitudes) the latter reduces to the former. In the next section the match with physical phenomena is discussed.

The idea is that the topological version of a theory is a useful device for defining perturbation theory in the topologically nontrivial sectors of the same theory? It allows one to separate in a convenient way the nonperturbative part, which is the integration over the instanton moduli space $\mathcal{M}$, from the perturbative part, described by the quantum fluctuations around the instanton 'vacuum'. At the same time, the topological theory can be recovered as a limit of the ordinary theory, when the quantum fluctuations are suppressed. The embedding of the former into the latter, which will be called topological embedding, is just a generalization of the usual procedure of treating the collective coordinates by "introducing 1" a la Faddeev-Popov 18, 19]: here I suggest to treat the topologically nontrivial sectors of a theory by "introducing the topological version" of the same theory. The observables of the topological theory are useful to define the (previously ill-defined) integration over collective coordinates. Of course, there are many inequivalent choices. One of them, the insertion of the volume form of the instanton moduli space $\mathcal{M}$, gives back the common result, in which the (infinite) volume of the moduli space appears as an overall factor. The other possibilities offered by the topological embedding and not contemplated within the usual approach, are indeed the insertions $\mathcal{M}$-top-forms made by products of topological observables $\mathcal{O}_{\gamma_{i}}$. In this approach, the infinite volume factor gets "regularized" and perturbation theory in the topologically nontrivial sectors is consistent.

In view of this, the amplitudes of the topological version of the theory give information about the nonperturbative nature of the complete theory. They are a useful device for extracting otherwise invisible properties of the instantonic configurations, that should have some physical meaning, if instantons do. Applying the results of ref. []] and the previous sections to QCD, it will be argued that the step amplitudes computed there and generalized here are related to a non abelian generalization of the Aharonov-Bohm effect.

\footnotetext{
${ }^{5}$ The 'classical' approach to this problem can be found, for example in [16, 17. The approach followed here is different under several aspects, but contains the old one.
} 
To be explicit, I focus on topological Yang-Mills theory with $G=S U(2), M=\mathbb{R}^{4}$ in the $k=1$ sector, in which case we have all the needed explicit formulæ. Let us decompose the gauge connection $A$ into $A_{0}+g A_{q}, A_{0}$ being the Belavin et al. instanton 13 and $A_{q}$ denoting the quantum fluctuation around it. Now, $A_{0}$ spans a moduli space $\mathcal{M}$, while $A_{q}$ is restricted to be perperdicular to it, otherwise tangential fluctuations are counted twice: once in $A_{0}$ and a second time in $A_{q}$. Having done this decomposition, we know that the integral over $A_{q}$ is well-defined. For example, in the quadratic approximantion, it gives the primed determinant of the kinetic operator, which has been computed explicitly by 't Hooft in ref. [14]. The $\mathcal{M}$ integral, on the other hand, is not well-defined. Usually [17, 16], one takes the attitude that the problem is due to perturbation theory and should disappear in the exact answer. This is not so useful from the practical point of view and is equivalent to declare that the perturbative expansion around instantons is inconsistent. Here I take a radically different attitude. The key idea is to say that there is a gauge-symmetry that has not been gauge-fixed before and that the ill-definition of the $\mathcal{M}$-integral is like the ill-definition of any functional integral before fixing ordinary geuge-symmetry. Indeed, there is no way, from the physical point of view, to privilege any $m \in \mathcal{M}$ (any position or size of the instanton, for example), because any $m$ is a minimum of the classical action. So, in the context of the perturbation expansion that I want to consider, deforming $m$ around $\mathcal{M}$ is a gauge-symmetry. It is exactly a topological symmetry: the most general continuous deformation of the instanton in the space of instantons. To cure the problem, we generalize the usual BRS recipe to global degrees of freedom. We have to do two things: i) to introduce ghosts (to be called topological ghosts) associated with the topological symmetry while preserving the nilpotence of the BRS operator; ii) to introduce a BRS closed operator that gauge-fixes the symmetry, making both the $\mathcal{M}$-integral and the integral over the topological ghosts meaningful. These aims can be achieved by embedding the topological version of the theory into the physical theory.

The topological ghosts are just the ghosts of topological Yang-Mills theory. So, let us write the BRST algebra of ordinary Yang-Mills theory

$$
s A^{a}=-D C^{a}, \quad s C^{a}=-\frac{1}{2} \varepsilon_{a b c} C^{b} C^{c},
$$

as the semidirect product of the BRST algebra of topological Yang-Mills theory

$$
\begin{array}{rlrl}
s A_{0 \mu}^{a} & =\psi_{0 \mu}^{a}+D_{\mu}\left(A_{0}\right) C_{0}^{a}, & s \psi_{0 \mu}^{a}=-D_{\mu} \phi_{0}^{a}-\varepsilon^{a}{ }_{b c} \psi_{0 \mu}^{b} C_{0}^{c}, \\
s \phi_{0}^{a}=\varepsilon^{a}{ }_{b c} \phi_{0}^{b} C_{0}^{c}, & s C_{0}^{a}=\phi_{0}^{a}-\frac{1}{2} \varepsilon^{a}{ }_{b c} C_{0}^{b} C_{0}^{c},
\end{array}
$$

times the following "remnant"

$$
\begin{aligned}
s A_{q \mu}^{a} & =-\frac{1}{g} \psi_{0 \mu}^{a}+D_{\mu}(A) C_{q}^{a}+\varepsilon_{b c}^{a} A_{q \mu}^{b} C_{0}^{c}, \\
s C_{q}^{a} & =-\frac{1}{g} \phi_{0}^{a}-\varepsilon^{a}{ }_{b c} C_{0}^{b} C_{q}^{c}-\frac{g}{2} \varepsilon^{a}{ }_{b c} C_{q}^{b} C_{q}^{c} .
\end{aligned}
$$

(4.1) is the sum of (4.2) and (4.3), $C^{a}$ being identified with $C_{0}^{a}+g C_{q}^{a}$. The complete functional integral is obtained by integrating over the 'topological fields' $A_{0}, \psi_{0}, C_{0}$ and $\phi_{0}$ and the 
quantum fluctuations $A_{q}$ and $C_{q}$. The functional measure over the topological fields reduces to $\int_{\mathcal{M}} d m d \hat{m}, m$ denoting the moduli and $\hat{m}$ being their ghost partners $(\hat{m}=s m)$. The topological subset (4.2) is closed under BRS transformations. This fact, as we know from [7], allows us to "solve the BRS algebra", i.e. find the explicit expressions of the topological ghosts that follow from nilpotence and the explicit expression of $A_{0}$. The complete solution is [7]

$$
\begin{aligned}
A_{0}^{a} & =\frac{2}{D} d x^{\mu} \eta_{\mu \nu}^{a}\left(x-x_{0}\right)^{\nu}, & C_{0}^{a} & =-\frac{2}{D} \hat{x}_{0}^{\mu} \eta_{\mu \nu}^{a}\left(x-x_{0}\right)^{\nu}, \\
\psi_{0 \mu}^{a} & =-4 \frac{\rho}{D^{2}} \eta_{\mu \nu}^{a}\left[\rho \hat{x}_{0}^{\nu}+\left(x-x_{0}\right)^{\nu} \hat{\rho}\right], & \phi_{0}^{a} & \left.=-\frac{2 \rho}{D^{2}} \eta_{\mu \nu}^{a}\left[\rho \hat{x}_{0}^{\mu}+2\left(x-x_{0}\right)^{\mu} \hat{\rho}\right)\right] \hat{x}_{0}^{\nu} .
\end{aligned}
$$

It is worth recalling that $\psi_{0}$ is gauge-fixed with the condition $D_{\mu}\left(A_{0}\right) \psi_{0 \mu}^{a}=0$. Finally, we can write the generating functional $Z\left[J_{q}\right]$ as

$$
Z\left[J_{q}\right]=\sum_{k \in \mathbb{Z}} \mathrm{e}^{i \theta k-\frac{8 \pi}{g^{2}}|k|} \int_{\mathcal{M}_{k}} d m Z_{k}\left[m, J_{q}\right],
$$

where $Z_{k}\left[m, J_{q}\right]$ is the partition function for a fixed value of the instanton moduli $m$ with instanton number $k$, namely

$$
Z_{k}\left[m, J_{q}\right]=\int d \hat{m} \int d A_{q} d C_{q} d \mu_{g f} \exp \left[-\mathcal{S}\left(A_{q}, m, \hat{m}\right)+\mathcal{S}_{g f}\left(A_{q}, m, \hat{m}\right)+J_{q} A_{q}\right] .
$$

$\mathcal{S}_{g f}\left(A_{q}, m, \hat{m}\right)$ denotes a suitable gauge-fixing term and $d \mu_{g f}$ is the relevant functional integration measure for Lagrange multipliers and antighosts. In this way, we have conveniently separated the nonperturbative and the perturbative aspects of the topologically nontrivial sectors of Yang-Mills theory. $Z\left[m, J_{q}\right]$ can be calculated perturbatively, since the background is fixed. The BRST algebra is (4.3) and the quantum fluctuations $A_{q}$ are restricted to be perpendicular to $\mathcal{M}$. They have a well-defined propagator and one can safely define Feynmann rules. The perturbative amplitude is in general $m$-dependent and the final amplitude is obtained after the (non-perturbative) integration over $\mathcal{M}$. The amplitudes computed in [7] and in the previous section are examples in which the full contribution comes from the nonperturbative part. Nevertheless, it is clear that they are also very peculiar amplitudes of ordinary Yang-Mills theory in the topologically nontrivial sectors.

The action $\mathcal{S}\left(A_{q}, m, \hat{m}\right)$ is obtained by expanding the usual Yang-Mills action around $A_{0}$ :

$$
\begin{aligned}
\mathcal{S}\left(A_{q}, m, \hat{m}\right) & =\frac{1}{4}\left[D_{\mu}\left(A_{0}\right) A_{q \nu}^{a}-D_{\nu}\left(A_{0}\right) A_{q \mu}^{a}\right]^{2}+\frac{1}{2} \varepsilon_{a b c} F_{\mu \nu}^{a}\left(A_{0}\right) A_{q \mu}^{b} A_{q \nu}^{c} \\
& +g \varepsilon_{a b c} A_{q \mu}^{a} A_{q \nu}^{b} D_{\mu}\left(A_{0}\right) A_{q \nu}^{c}+\frac{g^{2}}{4}\left(\varepsilon_{a b c} A_{q \mu}^{b} A_{q \nu}^{c}\right)^{2} .
\end{aligned}
$$

The gauge-fixing term is made by two pieces: the first term fixes the gauge symmetry $\delta A_{q \mu}^{a}=$ $D_{\mu} C_{q}^{a}$, while the second one fixes the topological symmetry. The first gauge-fixing is achieved with the usual condition $D_{\mu}\left(A_{0}\right) A_{q \mu}^{a}=0$, thus preserving the topological symmetry. So, the ordinary gauge-fixing term is

$$
\mathcal{S}_{g f}\left(A_{q}, m, \hat{m}\right)=\frac{1}{2}\left(D_{\mu}\left(A_{0}\right) A_{q \mu}^{a}\right)^{2}+\bar{C}_{q}^{a}\left[D_{\mu}\left(A_{0}\right) D_{\mu}(A) C_{q}^{a}+\varepsilon_{a b c} \psi_{0 \mu}^{b} A_{q \mu}^{c}+\varepsilon_{a b c} D_{\mu}\left(A_{0}\right) A_{q \mu}^{b} C_{0}^{c}\right] .
$$


The quadratic part $\mathcal{S}_{Q}$ of the gauge-fixed action $\mathcal{S}+\mathcal{S}_{g f}$ mixes $A_{q}$ and the ghosts $\bar{C}_{q}$ and $C_{q}$ in a nontrivial way: defining $V_{q}=\left[A_{q}, C_{q}, \bar{C}_{q}\right]$ and $Q(m, \hat{m})$ such that $\mathcal{S}_{Q}=V_{q}^{t} Q(m, \hat{m}) V_{q}$, the zero modes are collected in the vector $\left[\psi_{0}, \phi_{0}, 0\right]: Q(m, \hat{m})\left[\psi_{0}, \phi_{0}, 0\right]=0$.

Finally, let us discuss point ii) mentioned above, namely how to gauge-fix the topological symmetry. Usually, to gauge-fix a symmetry one introduces and operator of the form $\mathcal{O}=$ $\delta(\mathcal{G}) s \mathcal{G}, \mathcal{G}$ being the chosen gauge-fixing condition. $\mathcal{O}$ is clearly BRS closed and makes both the integration over the gauge-fields (via $\delta(\mathcal{G})$ ) and the integration over the gauge-ghosts (via $s \mathcal{G})$ meaningful. The moral of the story is that we have to introduce a BRS-closed operator $\mathcal{O}$ that makes both the $m$ - and $\hat{m}$-integrations meaningful. It is now clear that $\mathcal{O}$ has to be constructed with the topological observables $\mathcal{O}_{\gamma_{i}}$, i.e. $\mathcal{O}=\prod_{i} \mathcal{O}_{\gamma_{i}}$. Concretely, one can modify (4.6) into

$$
Z_{k}\left[m, J_{q}, \zeta\right]=\int d \hat{m} \int d A_{q} d C_{q} d \mu_{g f} \exp \left[-\mathcal{S}\left(A_{q}, m, \hat{m}\right)+\mathcal{S}_{g f}\left(A_{q}, m, \hat{m}\right)+J_{q} A_{q}+\zeta(\gamma) \mathcal{O}_{\gamma}\right],
$$

the term $\zeta(\gamma) \mathcal{O}_{\gamma}$ standing for all the possible insertions of topological observables. 't Hooft's choice [14], instead, dictated by a simple dimensional argument, is

$$
\mathcal{O}=\frac{\hat{\rho} \prod_{\mu=1}^{4}\left(\hat{x}_{0}\right)_{\mu}}{\rho^{5}}
$$

It does not fix the $x_{0}$-translations and leave the problem of the $\rho$-integration open.

Now, let us take a certain number of $\zeta$-functional derivatives in order to introduce a product of $\mathcal{O}_{\gamma_{i}}$ that saturates the moduli space dimension. Due to this, any term in $\mathcal{S}_{g f}$ containing $\psi_{0}$ or $C_{0}$ can be dropped and the action $\mathcal{S}+\mathcal{S}_{g f}$ reduces to the usual one. In the $g \rightarrow 0$ limit, only the quadratic part matters, which integrates to the primed determinants that combine with the $\mathrm{e}^{-\frac{8 \pi}{g^{2}}}$ factor to give a renormalization group invariant expression [14]. If we focus, for now, on dimensionless amplitudes (in some sense, the 'partition functions' of the topologically nontrivial sectors), i.e. amplitudes with no gluons $A_{q}$, this expression is just a constant 9 . Finally, the $\mathcal{M}$ integration gives back the topological amplitude associated to the $\mathcal{O}_{\gamma_{i}}$ 's, namely

$$
\text { const. } \mathrm{e}^{i \theta} \int_{\mathcal{M}} d m d \hat{m} \prod_{i}^{n} \mathcal{O}_{\gamma_{i}}(m, \hat{m}) \text {. }
$$

A choice like (4.11) 'renormalizes' the infinite factor that would be obtained with the 't Hooft measure (4.10). The (physically meaningful) freedom related to the choice of (4.11) can also be thought as the arbitrariness associated to the renormalization of the infinite volume factor. When there are gluons in the amplitude, (4.11) is just the measure over the moduli space. The amplitude has the form

$$
\text { const. } \mathrm{e}^{i \theta} \int_{\mathcal{M}} d m d \hat{m} \prod_{i}^{n} \mathcal{O}_{\gamma_{i}}(m, \hat{m}) \mathcal{A}\left(x_{1}, \ldots x_{n} ; m\right),
$$

\footnotetext{
${ }^{6}$ For more detailed explanations on how this happens and its implications, see section 2.1 of ref. 20].
} 
$\mathcal{A}\left(x_{1}, \ldots x_{n} ; m\right)$ denoting the perturbative amplitude at fixed $m$. It is clear that the measure (4.11) makes the $\mathcal{M}$-integral convergent. We conclude that the physical meaning of topological field theory is that it provides the set of consistent measures over the moduli space.

Due to the nature of the topological symmetry and its gauge-fixing, it is clear that the theory "depends" on the gauge-fixing itself, namely on the choice os $\gamma_{i}$ 's, in the sense that two gauge-fixings that are not continuously related to each other give different answers. It is very nontrivial, in general, to solve the problem of classifying the gauge-fixing dependence of a theory. Nevertheless, for the specific aspect of the problem that we are considering now, namely the dependence on the "gauge-fixing for collective coordinates" in the unit instanton number sector of $S U(2)$ (or $S U(N)$ ) Yang-Mills theory, we already know the answer, that is the multilink intersection theory elaborated $n$ the previous sections. To be explicit, the unlinking process described in section 2 is a non-continuous deformation whenever a complete multilink intersection is crossed. It is a continuous deformation for any incomplete intersection. That is why sometimes I speak about topological "gauge-fixing" and other times about "insertion of topological observables", when referring to this aspect of the theory. In other words, such a gauge-fixing choice is observable. Actually, our approach allows us to say that the topological aspects are the only genuine instantonic properties that can be observable. Dynamics only comes form the quantum fluctuations $A_{q}$, i.e. gluons propagating over the instanton background specified by $\prod_{i} \mathcal{O}_{\gamma_{i}}$.

A comment is in order about the regularization technique that is most convenient to treat perturbation theory around instantons (see also [14]). The dimensional technique presents some problems, since instantons are purely four dimensional objects. A Pauli-Villars-type technique seems to be better. To avoid the problem that the mass terms of the regulators break gaugeinvariance, one can use the following alternative regularization technique, firstly defined and used by Johansen in [21] within the usual perturbation theory framework. Embed Yang-Mills theory into $\mathrm{N}=4$ super Yang-Mills theory and break $\mathrm{N}=4$ supersymmetry down to $\mathrm{N}=0$ by giving mass terms to each additional field. For finite masses, the theory is finite and so is a good regulator. When one wants to recover the initial theory, one has to let the masses tend to infinity and the divergent terms have then to be subtracted with the usual renormalization algorithms.

To conclude, topological Yang-Mills theory is a useful device to define perturbation theory in the topologically nontrivial sectors of ordinary Yang-Mills theory and is also a certain limit of this theory itself. The topological amplidutes calculated so far can play a role in the "real theory".

\section{Non abelian analogue of the Aharonov-Bohm effect}

In this section, the relation between link invariants and physics is discussed, focusing, in particular on a non abelian analogue of the Aharonov-Bohm effect.

QCD is expected to confine and confinement is nonperturbative in nature. However, it is very difficult to get nonperturbative information about Yang-Mills theory. There are some non- 
perturbative aspects, nevertheless, (like instantons) that can be studied exactly, for example using the topological field theoretical device. These aspects of the theory could carry some, perhaps unconventional, sign that the theory confines and the link invariants could be interpreted as such a sign. The first thing that comes to one's mind when speaking about link invariants is the Aharonov-Bohm effect.

In Maxwell theory, a wire $\gamma$ of magnetic dipoles with magnetic moment $g$ per unit length (a thin circular solenoid, for example) generates a vector potential $\mathbf{A}(\mathbf{x}, \gamma)$ equal to

$$
\mathbf{A}\left(\mathbf{x}^{\prime}, \gamma\right)=-g \oint_{\gamma} d \mathbf{x} \wedge \nabla \frac{1}{\left|\mathbf{x}-\mathbf{x}^{\prime}\right|}
$$

so that the magnetic flux associated to a loop $\gamma^{\prime}$ is given by Gauss' formula (1.2):

$$
\Phi_{\gamma^{\prime}}\left(\mathbf{B}_{\gamma}\right)=\oint_{\gamma^{\prime}} \mathbf{A}\left(\mathbf{x}^{\prime}, \gamma\right) \cdot d \mathbf{x}^{\prime}=4 \pi g \gamma \chi \gamma^{\prime}
$$

The flux is quantized, according to the link number of the two loops. This is physically meaningful, since it can be observed (Aharonov-Bohm effect). The noticeable property of the field configuration is that both the electric and magnetic fields are zero everywhere except than within $\gamma$. So, in $\gamma$ there is a concentration of three entities: the gauge field, the positively charged matter and the negatively charged matter.

Quantum nonabelian gauge field theory should have the property that three (or more) entities like the above ones (i.e. the gauge bosons) are forced by the gauge symmetry to always screen one another. At least, this should be visible in any exact amplitude. Take, for example, $S U(2)$ gauge field theory and look at it as a $U(1)$ gauge theory (previliging some vector boson, which we call the photon) coupled to charged matter (the other two gauge bosons). Due to the underlying gauge symmetry, the "matter" and the "photon" are mixed together and indistinguishable. We know that link numbers appear in Maxwell theory plus matter only when the field strength and the charged matter are confined together. We also know, from the computations made in this paper, that link numbers in nonabelian gauge field theory appear naturally in special, but exact, amplitudes of the theory. That means precisely that, in the realm of the amplitudes here computed, which are the only exact nonperturbative amplitudes available so far, QCD, when regarded as a matter coupled QED, is such that the "matter" naturally screens the photon. Due to the underlying gauge symmetry, this holds for any "photon" and consequently for the entire group. In this sense, the link numbers that we have found can be regarded as an unconventional sign of confinement. A nontrivial consequence of this description is that, although QCD confines, the non-abelian analogue of the Aharonov-Bohm effect cannot be "screened" and should be experimentally observable. Maybe in some future we shall be able to construct wires carrying a nonvanishing color current and create something similar to the magnetic force lines that penetrate the superconductors [20]. Something like this should correspond to making experiments in a sector specified by (4.11).

Since confinement is expected to be a nonperturbative phenomenon, one should resum the perturbative series before being able to reveal it. In the perturbative framework, confinement is hardly visible, since when the coupling constant is zero the theory becomes practically abelian 
and an abelian theory does not confine. So, it may be very interesting to investigate some genuinely nonperturbative aspects of the theory like the ones considered in this paper. The amplitudes computed here represent special situations in which one is allowed to freeze the quantum fluctuations (so that no resummation at all is necessary) and yet get something nontrivial. Finally, it is clear that the topological embedding is an approach that also deserves to be studied per se.

Link numbers in QCD appear in the pure theory, i.e. they do not require the presence of matter. In section 3, we saw that they can also appear in presence of matter.

\section{Conclusions}

In ref. [7] and the present paper, new properties of the the Belavin-Polyakov-Schwarz-Tyupkin instanton were uncovered, using topological field theory as a tool. An unexpected connection with link theory came out. In [7] the presence of some link theory was detected, here the theory was identified completely. The feeling is that higher instanton numbers [11, 12] hide deeper mathematical concepts. No trace of vanishing amplitudes has been found so far, so one open problem (of the many) is to identify the mathematical meaning of the amplitudes with higher instanton number.

The second point concerns physics. The physical role of topological field theory was clarified by showing that its embedding in the associated physical theory is useful to perform the perturbative expansion in the topologically nontrivial sectors. The topological properties of very special solutions to the Yang-Mills field equations, like instantons are, are not expected to be unrelated to physics. Here they are related to a non abelian version of the Aharonov-Bohm effect.

\section{Acknowledgements}

I would like to thank V. Schomerus for many interesting discussions on 2-knots, link theory and related subjects. I also thank A. Johansen for discussions. This research was supported in part by the Packard Foundation and by NSF grant PHY-92-18167.

\section{References}

[1] D. Amati, K. Konishi, Y. Meurice, G.C. Rossi and G. Veneziano, Non-perturbative aspects in supersymmetric gauge-theories, Phys. Rep. 162 (1988) 169.

[2] E. Witten, Topological quantum field theories, Comm. Math. Phys. 117 (1988) 353.

[3] D. Anselmi and P. Frè, Twisted N=2 supergravity as topological gravity in four dimensions, Nucl. Phys. B392 (1993) 401.

[4] D. Anselmi and P. Frè, Topological twist in four dimensions, R-duality and hyperinstantons, Nucl. Phys. B404 (1993) 288. 
[5] A.S. Cattaneo, P. Cotta-Ramusino, A. Gamba and M. Martellini, The Donaldson-Witten invariants in pure 4D-QCD with order and disorder 't Hooft-like operators, preprint IFUM 493/FT and hepth/9502110.

[6] S.K. Donaldson, An application of gauge theories to the topology of four manifolds, J. Diff. Geom. 18 (1983) 269.

[7] D. Anselmi, Anomalies in instanton calculus, Nucl. Phys. B439 (1995) 617.

[8] D. Anselmi and P. Frè, Topological sigma models in four dimensions and triholomorphic maps, Nucl. Phys. B416 (1994) 255.

[9] D. Anselmi and P. Frè, Gauged hyperinstantons and monopole equations, Phys. Lett. 347B (1994) 247.

[10] L. Baulieu and I.M. Singer, Topological Yang-Mills theory, Nucl. Phys. B (proc. suppl.) 5 B (1988) 12.

[11] R. Jackiw, C. Nohl and C. Rebbi, Conformal properties of pseudoparticle configurations, Phys. Rev. D 15 (1977) 1642.

[12] M.F. Atiyah, N.J. Hitchin, V.G. Drinfeld and Yu.I. Manin, Construction of instantons, Phys. Lett. 65A (1978) 185.

[13] A.A. Belavin, A.M. Polyakov, A.S. Schwarz and Yu.S. Tyupkin, Pseudoparticle solutions of the Yang-Mills equations, Phys. Lett. 59B (1975) 85.

[14] G. 't Hooft, Computation of the quantum effects due to a four-dimensional pseudoparticle, Phys. Rev. D 14 (1976) 3432.

[15] E. Witten, Monopoles and four manifolds, Math. Research Lett. 1 (1994) 769.

[16] R. Rajaraman, Solitons and Instantons, North Holland, 1982.

[17] S. Coleman, Aspects of symmetry, Cambridge University Press, 1985.

[18] L.D. Faddeev and V.N. Popov, Phys. Lett. 25B (1967) 29.

[19] E. Gildener and A. Patrasciou, Pseudoparticle contributions to the energy spectrum of a one-dimensional system, Phys. Rev. D16 (1977) 423.

[20] D. Anselmi, On field theory quantization around instantons, preprint HUTP-95/A026, July 195.

[21] A.A. Johansen, Ultraviolet regularization of $\mathrm{N}=1$ supersymmetric theories by means of extended supersymmetries, Sov. J. Nucl. Phys. 45 (1987) 167. 